\title{
A Unifying Approach to Assessing Market Power in Deregulated Electricity Markets
}

\author{
Chenye $\mathrm{Wu}^{1}$, Subhonmesh Bose ${ }^{2}$, Adam Wierman ${ }^{2}$, and Hamed Mohesenian-Rad ${ }^{3}$ \\ ${ }^{1}$ Tsinghua University, ${ }^{2}$ California Institute of Technology, ${ }^{3}$ University of California, Riverside
}

\begin{abstract}
A competitive deregulated electricity market with increasingly active market players is foreseen to be the future of the electricity industry. In such settings, market power assessment is a primary concern. In this paper, we propose a novel functional approach for measuring long term market power that unifies a variety of popular market power indices. Specifically, the new measure, termed transmission constrained network flow (TCNF), unifies three large classes of market power measures: residual supply based, network flow based, and minimal generation based. Further, TCNF provides valuable information about market power not captured by prior indices. We derive its analytic properties and test its efficacy on IEEE test systems.
\end{abstract}

Index Terms-Market power, electricity markets.

\section{INTRODUCTION}

Market monitoring is essential for maintaining efficient electricity markets: not only is market power a significant challenge to the efficiency of today's electricity markets, e.g., the California energy crisis of 2000; monitoring market power will become even more important in the coming years as we move toward a future smart grid that includes significant penetration of renewable energy, distributed generation, energy storage, and increased penetration of demand-response programs. As a result, new market power measures that quantify the impact of these emerging developments are crucial.

However, electricity markets are particularly difficult environments in which to identify market power. In fact, regulators and economists have yet to develop standardized market power measures for the electricity market, and the measures that are in use today are typically ad hoc. As a result, there is a growing (but typically fractured) literature seeking to improve market power analysis for electricity markets.

In particular, there are two almost completely distinct literatures that focus on "long-term analysis" and "short-term analysis". Long term approaches most often study the potential for market power, i.e., they are usually ex-ante. They are useful for tasks such as market design evaluation, merger analysis, operation planning, as well as for identifying "mustrun" generators in advance. Short term approaches most often study the exploitation of market power, i.e., they are usually ex-post. Typically, they are applied close to the spot market and focus on things such as immediately mitigating market conduct via penalties for withholding generation.

Emails: wucy05@mails.thu.edu.cn, bosesecaltech.edu, adamw@caltech.edu, hamed@ee.ucr.edu. This work was supported in part by the NSF under NetSE grant CNS 0911041, ECCS 1253516, ARPAE grant DE-AR0000226, Southern California Edison, Nat. Sc. Council of Taiwan, R.O.C. grant NSC 101-3113-P-008-001, Resnick Inst., Okawa Found., Nat. Basic Research Prog. of China Grant 2011CBA00300, 2011 CBA00301.
In this paper, we focus on long term market power analysis; however even within this domain the literature is quite fractured. Broadly, these measures can be divided into three classes that we term: "network flow based", e.g., [1], [2], "residual supply based", e.g., [3], [4], and "minimal generation based", e.g., [5], [6]. We introduce each of these in Section II.

Given the fractured state of the market power literature, it is important to begin to add "structure" by characterizing the relationship between the existing measures. To that end, the goal of this paper is to develop a unified approach for measuring long term market power that bridges these three types of market power analysis. Specifically, our main contribution is to introduce a new functional market power measure, termed "transmission constrained network flow" (TCNF) which unifies network flow based, residual supply based, and minimum generation based market power measures. In fact, the single-value indices in these three measures can be seen as special cases of the new functional measure. Further, TCNF can provide valuable information about market power not captured by prior indices. For example, the slope of the TCNF function assesses the importance of each generator at various demand or renewable generation levels. Finally, calculating TCNF is computationally easy, which makes it an attractive measure for market power analysis of large networks in practice.

\section{MARKeT POWER MEASURES}

Market power is most often defined as the ability to profitably alter prices away from competitive levels [7]-[10]. Thus, market power coincides with a form of market "dominance", where a player has the power to behave independently of competitors and consumers in a manner that increases its profitability. Such dominance can be gained globally, e.g., by a power supplier with a large enough generation capacity in a power pool, or locally, e.g., by a power supplier in a region which has limited ability to import less expensive electricity through transmission lines [11].

While the measures used in practice today are mostly ad hoc, in recent years, principled design of market power measures has begun to emerge, e.g., see [9] for an excellent survey. When discussing such measures, a crucial distinction is whether they focus on long term or short term analysis.

In this paper, we focus on long term market power analysis. There is a large literature focusing on long term market power analysis. Some examples of measures for long-term analysis are [1], [3], [12]-[17]. Many of the earliest suggested measures of market power ignore transmission constraints. For example, Bushnell et al. introduced the pivotal supplier index (PSI) as a binary indicator examining whether the capacity of a 
generator is larger than the supply surplus, i.e., the difference between the total supply and the total demand [12]. Later, Sheffrin et al. refined PSI by measuring market power on a continuous scale, and proposed the residual supply index (RSI) in [13], [14]. This index is used by the California ISO to assure price competitiveness [15]. The electric reliability council of Texas (ERCOT) uses a different measure, the element competitiveness index (ECI) [16], which is based on the Herfindahl-Hirschmann index (HHI) [17].

Each of these indices measure market power purely in terms of dominance in generation capacity and fail to consider the impact of transmission constraints, which are a crucial component of market power in electricity markets because they can create situations where a power supplier has significant local market power if the region has limited ability to import less expensive electricity through transmission lines.

More recently, a variety of measures that include transmission constraints have been introduced. We can classify these into three categories that we term: "residual supply based", "network flow based", and "minimal generation based".

\section{A. Residual supply based measures}

Residual supply based measures are exemplified by [3], [4], which propose the transmission-constrained residual supply index (TCRSI). For each generator $s$, TCRSI measures the maximum total load that the transmission-constrained electricity market can support if generator of interest, $s$, is excluded:

$$
\begin{aligned}
\operatorname{TCRSI}_{s}=\underset{q, t}{\operatorname{maximize}} & t \\
\text { subject to } & \mathbf{1}^{\dagger} q=\mathbf{1}^{\dagger}(\bar{d} t), \\
& -b \leq H_{q} q-H_{d}(\bar{d} t) \leq b, \\
& q_{s}=0, \quad 0 \leq q_{i} \leq \bar{q}_{i}, i \neq s .
\end{aligned}
$$

where $q$ is the supply vector, $t$ is the demand scaling parameter, $H_{q}$ is the generation shift factor matrix, $H_{d}$ is load shift factor matrix, $b$ is the transmission line capacity vector, $\overline{q_{i}}$ is the capacity of generator $i, \bar{d}_{j}$ is the demand of load $j, \mathbf{1}$ is a unit vector, and $\dagger$ denotes transposition. If $\mathrm{TCRSI}_{s}<1$, then generator $s$ may gain market power. Figure 1 gives an example for TCRSI. For $G_{1}$, TCRSI is $3.2 / 7$, the fraction of demand that can be met with available supply.

\section{B. Network flow based measures}

Network flow based measures are exemplified by [1], [2], which model market power in the presence of transmission constraints in terms of the maximal network flow (MNF) achievable without the generator of interest. Thus, the measures in [1], [2] are similar to TCRSI in motivation; however, they do not use power flow equations, which are crucial to understanding power systems. To highlight this fact, note that a key result in [1], [2] is that market power is supermodular, i.e., there is always an incentive for generators to collude. However, this conclusion no longer holds if the power flow respects the impedances, e.g., in the network in Figure 1. Also, see Section V for a counterexample in IEEE test systems.

\section{Minimal generation based measures}

In the above two definitions of market power, the fraction of unmet demand is measured when a generator at bus $s$ is not in service. Alternatively, we can assess the market power of this generator by calculating the minimum generation required from it to meet the total demand. In particular, minimal generation based measures typically focus on "must run generators", e.g., [5], [6]. They come in many forms, but are exemplified by the transmission-constrained minimal generator index (TCMGI):

$$
\begin{aligned}
\operatorname{TCMGI}_{s}=\underset{q}{\operatorname{minimize}} & q_{s} \\
\text { subject to } & \mathbf{1}^{\dagger} q=\mathbf{1}^{\dagger} \bar{d}, \\
& -b \leq H_{q} q-H_{d} \bar{d} \leq b, \\
& 0 \leq q_{i} \leq \bar{q}_{i} .
\end{aligned}
$$

Unlike in (1), where $q_{s}=0$ is a constant and the total load scaling factor $t$ is a variable, in (2) the output of generator $s$ is a variable and the total load is a constant. If $\mathrm{TCMGI}_{s}>0$, then generator $s$ may gain market power.

Note that $\mathrm{TCMGI}_{s}$ does not, in general, equal the shortfall in the network when generator at bus $s$ is not operational. To illustrate this, consider the network in Figure 1. It can be checked that $\mathrm{TCMGI}_{1}=4.2 p u$ while the shortfall is actually $3.8 p u$ when the same generator is not in service. The shortfall and the minimum generation are, however, related. We explore this in more detail in the next section.

\section{A UNIFYING MARKET POWER FUNCTIONAL}

The brief background on long term market power measures in the previous section highlights the wide variety of measures that exist, and that the literature is very fractured. The goal of this paper is to provide a unified approach that facilitates the study of future grid phenomena.

Toward that goal, we propose a new "functional" measure for market power that unifies the residual supply based measures, network flow based measures, and minimal generation based measures. We term our measure the transmissionconstrained network flow (TCNF), and define it as follows:

$$
\begin{aligned}
\operatorname{TCNF}_{s}(\rho)=\underset{q, d}{\operatorname{maximize}} & D \\
\text { subject to } & \mathbf{1}^{\dagger} q=\mathbf{1}^{\dagger} d=D, \\
& -b \leq H_{g} q-H_{d} d \leq b, \\
& 0 \leq q_{i} \leq \bar{q}_{i}, \\
& q_{s} \leq \rho, \\
& 0 \leq d_{j} \leq \bar{d}_{j} .
\end{aligned}
$$

It is immediately clear that $\operatorname{TCNF}_{s}(\rho)$ generalizes network flow based and residual supply based measures. In particular, $\mathrm{TCNF}_{s}(0)$ is the maximal network flow satisfying the DC power flow constraints when generator $s$ is removed. Further, $\mathrm{TCNF}_{s}(0)$ is nearly the same as $\mathrm{TCRSI}_{s}$, with the caveat that the load is not restricted to be scaled uniformly at different buses. The relationship to minimal generation measures is not as clear. However, note that $\mathrm{TCNF}_{s}\left(\mathrm{TCMGI}_{s}\right)=\mathbf{1}^{\dagger} \bar{d}$. Further, we can prove that $\operatorname{TCNF}_{s}(\rho)$ is the inverse of a generalization of $\mathrm{TCMGI}_{s}$. To see this, next we define the transmission- 


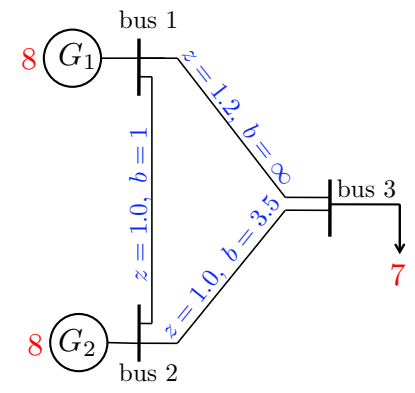

Fig. 1. A small network to illustrate market power indices. All quantities are measured in per units (p.u.). $z$ denotes impedance and $b$ denotes line capacity.

constrained minimal generation (TCMG) function as:

$$
\begin{aligned}
\operatorname{TCMG}_{s}(D)=\underset{q, d}{\operatorname{minimize}} & q_{s}, \\
\text { subject to } & \mathbf{1}^{\dagger} q=\mathbf{1}^{\dagger} d=D, \\
& -b \leq H_{g} q-H_{d} d \leq b, \\
& 0 \leq q_{i} \leq \bar{q}_{i}, \\
& 0 \leq d_{j} \leq \bar{d}_{j} .
\end{aligned}
$$

Note that, $\mathrm{TCMG}_{s}\left(\mathbf{1}^{\dagger} \bar{d}\right)$ is the same as $\mathrm{TCMGI}_{s}$. Further, the function $\mathrm{TCMG}_{s}(D)$ generalizes $\mathrm{TCMGI}_{s}$ in the sense that it measures the minimum generation that is needed from generator $s$ to support load $0 \leq D<1^{\dagger} \bar{d}$, i.e., a portion of the total load. Using this measure, we can state the following theorem. The proof is omitted due to space constraints.

Theorem 1. For each generator $s$, the $\operatorname{TCNF}_{s}(\rho)$ and $\mathrm{TCMG}_{s}(D)$ functions are inverse functions of each other, i.e., for $0 \leq D \leq \mathbf{1}^{\dagger} \bar{d}$, TCNF $\left[\operatorname{TCMG}_{s}(D)\right]=D$. Furthermore, $\operatorname{TCNF}_{s}(\rho)$ is a continuous, piecewise linear, concave, and monotonic increasing and $\mathrm{TCMG}_{s}(D)$ is a continuous, piecewise linear, convex, and monotonic increasing functions.

Thus, $\operatorname{TCNF}_{s}(\rho)$ unifies the three classes of long term market power measures. A key distinction of $\operatorname{TCNF}_{s}(\rho)$ from earlier measures is its functional form. By providing a parameterized definition of market power, it allows us to study the impact of the generator on the satisfiable demand $(D)$. In other words, it captures the necessary capacity from generator $s$ in order to satisfy different levels of demand, which is crucial when seeking to understand market power in the presence of dispatchable loads, demand response, and distributed and renewable generation. The case studies in Section IV highlight the usefulness of the functional form for these settings.

\section{A. A simple example}

Before discussing additional properties of TCNF, we present a simple example of its use on the network shown in Figure 1. Recall that we have earlier calculated TCRSI and TCMGI for the network in Figure 1.

In Figure 2 we plot the TCNF function for each of the two generators in the network shown in Figure 1. As stated in Theorem 1, the TCNF function is piecewise linear, increasing, and concave for both generators. Additionally, the $y$-intercept matches the TCRSI for each generator and the quantity at which the maximal demand is satisfied matches the TCMGI for each generator. Further, we can interpret the graph as

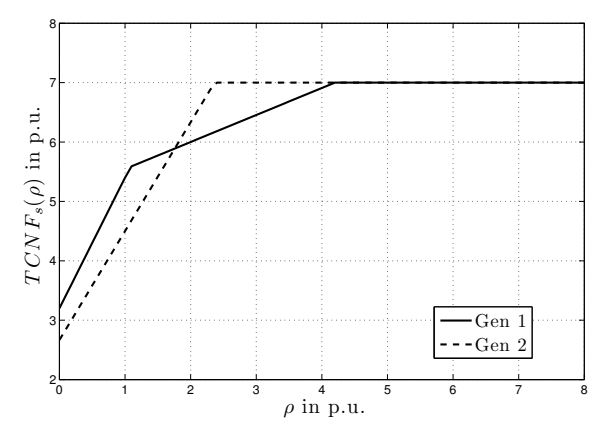

Fig. 2. TCNF for each generator in the network shown in Figure 1.

follows. Consider a level of total demand of $6.5 p u$ that is less than the target level of $7 p u$. At this demand level, slope of $\mathrm{TCNF}_{1}$ is lower than that of $\mathrm{TCNF}_{2}$, i.e., $G_{2}$ has to supply more than $G_{1}$ to satisfy an extra unit of demand and hence $G_{2}$ is more valuable to the system operator at this demand level. For a system with dispatchable loads, the plots of $\mathrm{TCNF}_{s}$ as in Figure 2 gives valuable insight into the importance of each generator at various demand levels.

Alternatively, consider the case where one generator is not operational, i.e., consider $\operatorname{TCNF}_{1}(0)$ and $\mathrm{TCNF}_{2}(0)$ in Figure 2. The shortfall in demand is higher when $G_{2}$ is not operational and hence is more valuable to the system operator from a security standpoint. We can generalize this interpretation to any generation level $\rho$. In other words, generator at bus $s$ with the minimum $\operatorname{TCNF}_{s}(\rho)$ is more valuable and hence enjoys more market power. In Figure 2, notice that the generator with most market power changes with the generation level $\rho$.

The discussion above highlights the valuable extra information provided as a result of the functional form of TCNF.

\section{B. Computation of TCNF}

It is important to discuss briefly the computational demands of calculating the TCNF. In particular, computing the solution to the optimal power flow problem with the lossless DC-OPF approximation is computationally expensive for large power networks [18], [19]. Since TCNF is a variation of the OPF problem, computing it for generator at bus $s$ for all $0 \leq \rho \leq \bar{q}_{s}$ may also be expected to be computationally expensive.

However, TCNF has a number of properties that can be exploited to simplify its computational demands. Importantly, the function $\operatorname{TCNF}_{s}(\rho)$ is piecewise linear. Thus, using Lagrangian duality [20], [21], we can characterize the slopes of the piecewise linear segments of $\operatorname{TCNF}_{s}(\rho)$ and use these slopes to provide an efficient way to compute $\mathrm{TCNF}_{s}$.

More specifically, for generator $s$, let the Lagrange multiplier for constraint $q_{s} \leq \rho$ in (3) be $\mu$. For any function $f(z)$ in variable $z$, define $(d f(z) / d z)^{+}$as its right-hand derivative [22]. Also, for any variable $z$ in the problem, let $z^{*}$ denote its value at the optimum. We can now relate the slopes of the linear segments of the functions $\operatorname{TCNF}_{s}(\rho)$ as follows:

$$
\left(\frac{d \operatorname{TCNF}_{s}(\rho)}{d \rho}\right)^{+}=\mu^{*} .
$$

The proof leading to (5) is omitted due to space constraints. Note that, since $\operatorname{TCNF}_{s}(\rho)$ is piecewise linear it is non- 
differentiable at the end-points of each line segment. But, (5) only involves the right-hand derivative and is well-defined.

Next, using (5), we present the following recursive algorithm for computing $\operatorname{TCNF}_{s}(\rho)$ for $\rho$ in any interval $[a, b]$.

\section{Algorithm 1.}

1) Compute $T C N F_{s}(a)$ and $T C N F_{s}(b)$ using a standard primal-dual linear program solver [21], [23]. Also, obtain the Lagrange multiplier $\mu^{*}$ (call them $\mu^{*}(a)$ and $\mu^{*}(b)$ respectively). Let $\left(\rho_{a, b}, D_{a, b}\right)$ solve:

$$
\begin{aligned}
D_{a, b}-\operatorname{TCNF}_{s}(a) & =\mu^{*}(a)\left[\rho_{a, b}-a\right], \\
D_{a, b}-\operatorname{TCNF}_{s}(b) & =\mu^{*}(b)\left[\rho_{a, b}-b\right],
\end{aligned}
$$

where $a \leq \rho_{a, b} \leq b$.

2) If $\operatorname{TCNF}_{s}\left(\rho_{a, b}\right)=D_{a, b}$, then in interval $[a, b]$ :

$\operatorname{TCNF}_{s}(\rho)= \begin{cases}\operatorname{TCNF}_{s}(a)+\mu^{*}(a)[\rho-a], & a \leq \rho \leq \rho_{a, b}, \\ \operatorname{TCNF}_{s}(b)+\mu^{*}(b)[\rho-b], & \rho_{a, b} \leq \rho \leq b .\end{cases}$

If $\operatorname{TCNF}_{s}\left(\rho_{a, b}\right) \neq D_{a, b}$, then repeat steps 1 and 2 over the intervals $\left[a, \rho_{a, b}\right]$ and $\left[\rho_{a, b}, b\right]$.

Using Algorithm 1 we can compute $\operatorname{TCNF}_{s}(\rho)$ for $\rho$ in $\left[0, \bar{q}_{s}\right]$. Further, it can be checked that the algorithm finishes in at most $2 \omega+1$ iterations, where $\omega$ is the number of line segments in $\operatorname{TCNF}_{s}(\rho)$ over $\rho$ in $\left[0, \bar{q}_{s}\right]$. Also, $\omega \leq 2^{E}$, where $E$ denotes the number of transmission lines in the grid. In practice, however, the number of segments is usually quite small and so this method computes $\operatorname{TCNF}_{s}(\rho)$ efficiently. This can be observed empirically for the case studies in Section IV.

\section{CAse Studies}

In order to illustrate the use of TCNF in realistic scenarios, we explore the market power of generators in the IEEE test systems in [24]. This exploration highlights the importance of market power, and the subtleties of the interaction of market power with varying demand (e.g., as a result of distributed renewable generation) and dispatchable load.

We have explored TCNF in the context of all the IEEE test systems, but here we only discuss two cases: the IEEE 9-bus system and the IEEE 39-bus system. In each case, we look at a variety of scalings of the target demands in the test system to understand the impact of demand fluctuations and distributed renewable generation. Specifically, the target demands are all scaled uniformly by a scalar $t \geq 0$. Further, in most IEEE systems, generators have a minimum level of generation, i.e., $q \geq q$. We modify $q=\mathbf{0}$ for convenience.

The results for the 9-bus system (with $t=1.8$ and 2.0) and 39-bus system (with $t=1.0$ and 1.1) are shown in Figure 3 (a) and 3(b) respectively. For the 39 bus system, $\operatorname{TCNF}_{s}(\rho)$ for only the generators at buses $31,35,38$ and 39 is plotted in order to simplify the presentation. Before discussing each case in detail, note that for all plots, the $y$-intercept of each $\mathrm{TCNF}_{s}$ plot is the $\mathrm{TCRSI}_{s}$ and the minimum $\rho$ for which all demands are met is $\mathrm{TCMGI}_{s}$.

\section{A. IEEE 9-bus system}

The most important point to observe in the 9-bus system is that for $t=1.8$, the order of generators according to market power (or $\mathrm{TCNF}_{s}$ ) changes with $\rho$. This indicates that incremental value of each firm to meet additional demand will typically depend on the actual demand, and thus the the quantity of dispatchable load interacts strongly with the market power of generators. This effect was not observable using previous market power measures. Further, Figure 3(a) highlights that generator 2 has the highest market power when $t=1.8$, while generator 3 has the most market power when $t=2.0$. Since the demand levels vary over a typical day, generators can be expected to have their market power vary during the day as a result of, e.g., the amount of distributed renewable generation available. This can play a significant role in the strategic behavior of generators. The plot in Figure 4(a) illustrates this effect further. It shows the lower envelope of the TCNF curves for the generators listed in the legend, and uses color to indicate which generator defines this lower envelope, i.e., which generator has the most market power. The figure highlights that there is a subtle interaction between fluctuating demand, dispatchable load, and market power.

\section{B. IEEE 39-bus system}

The observations about the 39-bus system parallel those above for the 9-bus system. In particular, we can see that generator 39 maintains the highest market power for the scaling of $t=1.0$ and $t=1.1$. However, the ordering of market power changes, e.g., when $t=1.0$, generator at bus 38 is not pivotal but gains significant market power for $t=1.1$ especially at higher $\rho$. To illustrate the role of demand variation, we only consider generators at buses 31,35 and 38 and plot the lower envelope of the $\mathrm{TCNF}_{s}$ for these generators for demand levels ranging from $t=1.0$ to $t=1.15$ in Figure 4(b). It is interesting that the generator with highest market power varies a significantly depending on the amount of dispatchable load considered and the variation in demand. We emphasize that the nuances of such a complex interactions can not be observed using any of the prior market power measures considered in the literature.

\section{CONCLUding REMARKS}

In this paper we have introduced a new long term market power measure that unifies three large, distinct classes of measures considered previously in the literature. This new transmission constrained network flow (TCNF) functional measure, not only unifies prior measures, it also provides new detailed insight into the market power of generators. To highlight this, we have performed case studies using the IEEE test systems.

This paper is a first step toward understanding and developing the TCNF measure. There are a number of future directions that are important to study. For example, it would be interesting to incorporate an $\mathrm{AC}$ power flow model in place of the DC model considered in this paper. Further, an important generalization is to generation "firms" with multiple generators. We have briefly looked at this issue already, and have found it to be subtle and important. In particular, previous work has suggested that market power is supermodular, and thus there is always incentives for generators to collude and form large firms [1], [2]. However, [1], [2] did not use power flow constraints in their study, and we have found using TCNF that, while often true, there are certainly counter examples 


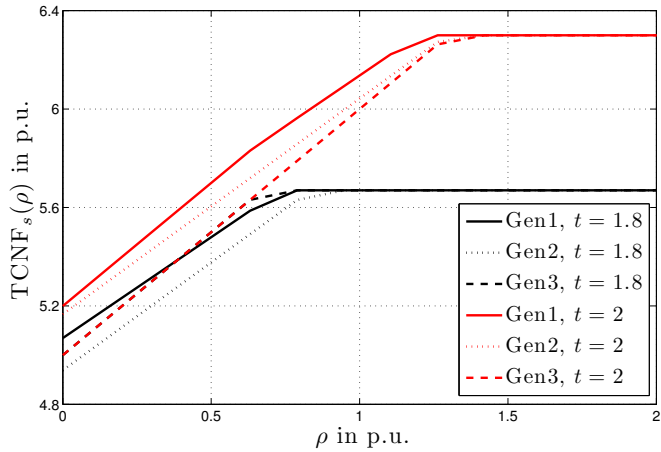

(a) IEEE 9-bus system

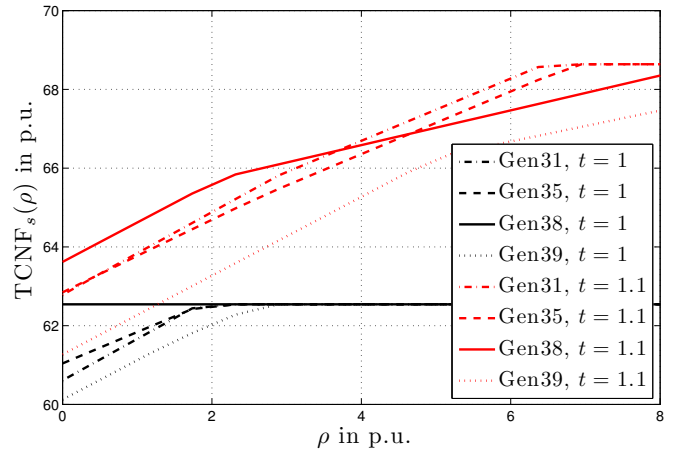

(b) IEEE 39-bus system

Fig. 3. TCNF for various generators in the IEEE 9-bus and 39-bus benchmark systems.

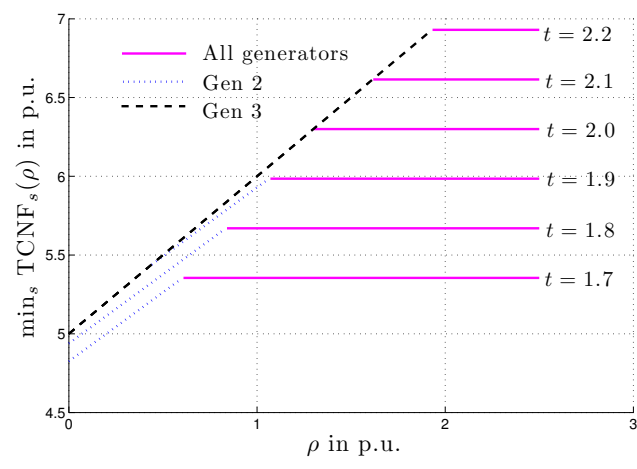

(a) IEEE 9-bus system

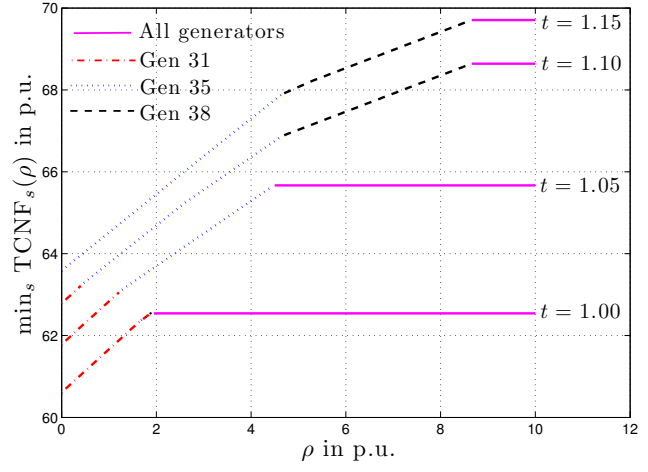

(b) IEEE 39-bus system

Fig. 4. The lower envelope of $\mathrm{TCNF}_{s}$ for the listed generators in the IEEE 9-bus and 39-bus benchmark systems with different scalings of demand.

to such a claim. For example, in the IEEE 9-bus system with $t=1.7$, supermodularity does not hold, e.g., for the combination of generators at buses $(1,3)$ and $(2,3)$.

\section{REFERENCES}

[1] J. Chen, M. Macauley, and A. Marathe, "Network topology and locational market power," Computational Econ., vol. 34, pp. 21-35, 2009.

[2] K. Atkins, J. Chen, V. S. A. Kumar, M. Macauley, and A. Marathe, "Locational market power in network constrained markets," J. of Economic Behavior \& Organization, vol. 70, no. 1, pp. 416-430, May 2009.

[3] Y. Y. Lee, R. Baldick, and J. Hur, "Firm-based measurements of market power in transmission-constrained electricity markets," IEEE Trans. on Power Systems, vol. 26, no. 4, pp. 1962-1970, Nov. 2011.

[4] L. Xu and R. Baldick, "Transmission-constrained residual demand derivative in electricity markets," IEEE Transactions on Power Systems, vol. 22 , no. 4, pp. 1563-1573, Nov. 2007.

[5] D. Gan and D. Bourcier, "Locational market power screening and congestion management: experience and suggestions," IEEE Transactions on Power Systems, vol. 17, no. 1, pp. 180-185, 2002.

[6] P. Wang, Y. Xiao, and Y. Ding, "Nodal market power assessment in electricity markets," IEEE Trans. on Pow. Sys., vol. 19, no. 3, pp. 13731379, 2004.

[7] S. Stoft, "Power system economics: Designing market for power," 2002.

[8] A. Mas-Colell, M. Whinston, J. Green et al., Microeconomic theory. New York: Oxford university press, 1995, vol. 1.

[9] P. Twomey, R. Green, K. Neuhoff, and D. Newbery, "A review of the monitoring of market power the possible roles of tsos in monitoring for market power issues in congested transmission systems," 2006.

[10] P. Burns, M. Huggins, R. Lydon, and et al., Generators' Strategies in the England and Wales Electricity Market: A Synthesis of Simulation Modelling and Econometric Analysis, ser. Technical paper. University of Bath, 2004. [Online]. Available: http://books.google.com/books?id= - tj7PAAACAAJ

[11] A. K. David and F. Wen, "Market power in electricity supply," IEEE Trans. on Energy Conversion, vol. 16, no. 4, pp. 352-360, Dec. 2001.
[12] J. Bushnell and C. Day, "An international comparison of models for measuring market power in electricity," Energy Modeling Forum Stanford University, 1999.

[13] A. Y. Sheffrin and J. Chen, "Predicting market power in wholesale electricity markets," in Proc. of the Western Conference of the Advances in Regulation and Competition, South Lake Tahoe, 2002.

[14] A. Y. Sheffrin, J. Chen, and B. F. Hobbs, "Watching watts to prevent abuse of power," IEEE Power and Energy Magazine, vol. 2.

[15] California Independent System Operator, "Market power and competitiveness," In Annual Report on Market Issues and Performance, http://www.caiso.com/1c5f/1c5fbe6a1a720.html, 1998.

[16] Electric Reliability Council of Texas, "Ercot protocols," http://www.ercot.com, 2001.

[17] R. Schmalensee and B. W. Golub, "Estimating effective concentration in deregulated wholesale electricity markets," RAND Journal of Economics, vol. 15 , no. 1 , pp. 12-26, Mar. 1984

[18] K. Purchala, L. Meeus, D. Van Dommelen, and R. Belmans, "Usefulness of DC power flow for active power flow analysis," in Proc. of IEEE PES General Meeting. IEEE, 2005, pp. 2457-2462.

[19] K. S. Pandya and S. K. Joshi, "A survey of optimal power flow methods," J. of Theo. and Applied Info. Tech., vol. 4, no. 5, pp. 450-458, 2008.

[20] A. Holder, Parametirc LP Analysis. John Wiley \& Sons, 2010.

[21] S. Boyd and L. Vandenberghe, Convex Optimization. Cambridge University Press, 2004

[22] R. M. Freund, "Postoptimal analysis of a linear program under simultaneous changes in matrix coefficients," in Mathematical Programming Essays in Honor of George B. Dantzig Part I, ser. Mathematical Programming Studies. Springer Berlin Heidelberg, 1985, pp. 1-13.

[23] R. Monteiro and I. Adler, "Interior path following primal-dual algorithms. part i: Linear programming," Mathematical programming, vol. 44, no. 1, pp. 27-41, 1989.

[24] "IEEE Power Systems Test Case Archive," http://www.ee.washington.edu/research/pstca. 